\title{
Ne confondons pas coquilles et coquillages
}

Vision diachronique de l'archéologie des mollusques le long de la façade atlantique

Do not mix up shells and shellfish. A diachronic archaeological view of molluscs along the Alantic facade

\section{Catherine Dupont}

\section{(2) OpenEdition}

Journals

Édition électronique

URL : https://journals.openedition.org/tc/6685

DOI : $10.4000 /$ tc. 6685

ISSN : 1952-420X

Éditeur

Éditions de l'EHESS

Édition imprimée

Date de publication : 15 décembre 2012

Pagination : 242-259

ISBN : 978-2-7351-1534-1

ISSN : 0248-6016

\section{Référence électronique}

Catherine Dupont, « Ne confondons pas coquilles et coquillages», Techniques \& Culture [En ligne], 59 |

2012, mis en ligne le 15 décembre 2015, consulté le 29 septembre 2022. URL : http://

journals.openedition.org/tc/6685; DOI : https://doi.org/10.4000/tc.6685 


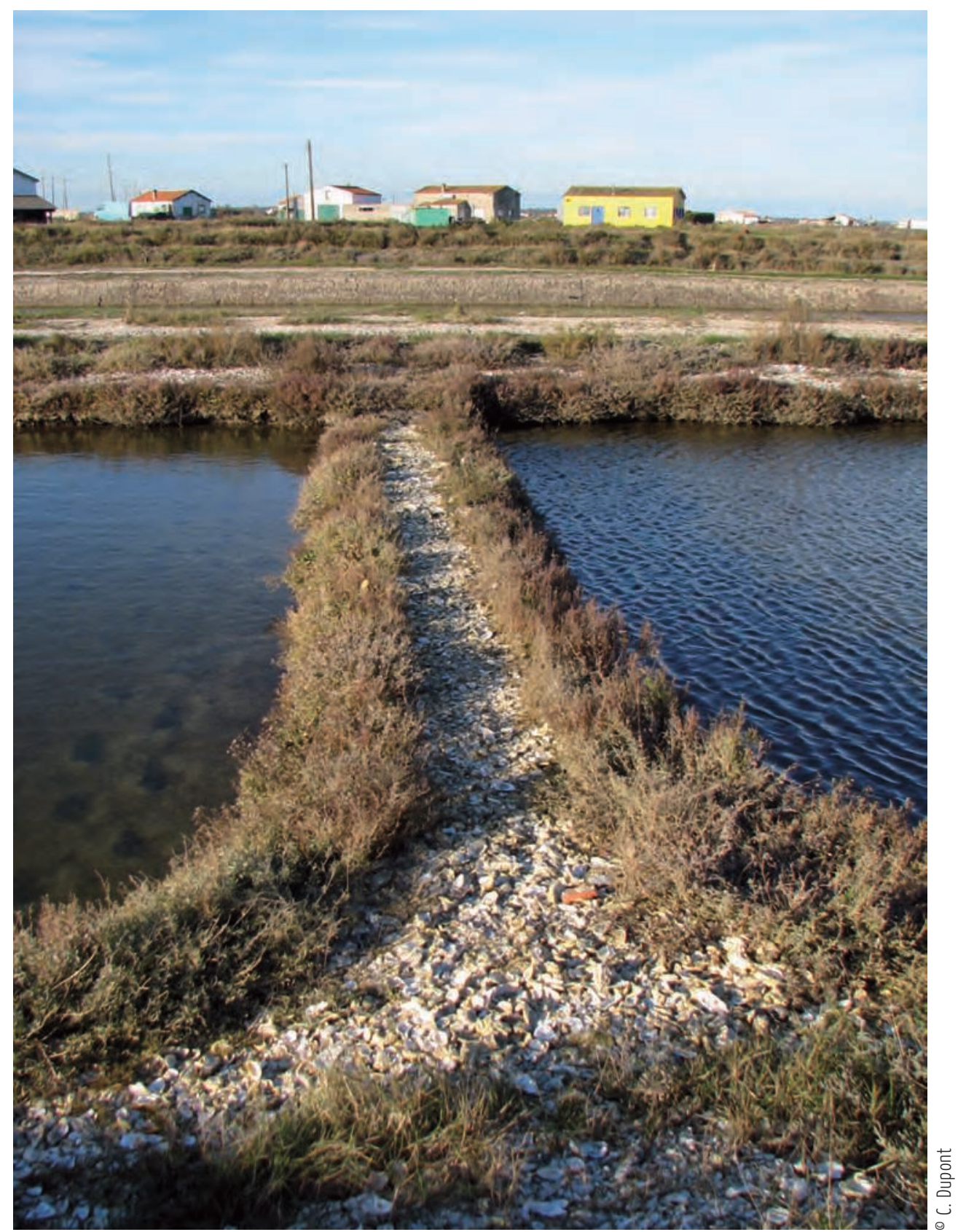




\section{NE CONFONDONS PAS COQUIILLES ET COQUILLAGES}

\section{Vision diachronique de l'archéologie des mollusques le long de la façade atlantique}

La rareté des sites archéologiques du Paléolithique associés à des déchets alimentaires coquilliers s'oppose à la multiplication de ce type de vestiges au Mésolithique le long du littoral atlantique européen (Gutiérrez-Zugasti et al. 2011). Cette simple observation fait que régulièrement le Mésolithique est cité comme une période durant laquelle l'exploitation des ressources marines en tant que ressources alimentaires est une activité qui s'intensifie (Giot et al. 1998; Garanger 1992). Cette vision tronquée de notre connaissance de l'exploitation des mollusques marins est calquée sur un des biais majeurs de l'archéologie, celui de ne pouvoir observer que ce qui a été préservé jusqu'à nos jours ou que ce qui est accessible par les moyens de recherche actuels (Kayser 1989; Dupont 2006). Ainsi, sur la côte atlantique française, les plus anciens dépotoirs coquilliers connus sont ceux de la fin du Mésolithique datés du vile millénaire. Les amas coquilliers précédents sont soit immergés, soit ensevelis sous des sédiments meubles ou ont été détruits par les phénomènes érosifs.

Les hommes du Paléolithique avaient pourtant accès à la mer. En témoignent les coquilles utilisées en parure, découvertes régulièrement dans les sépultures (Taborin 1993). Cette différence de représentativité archéologique du monde marin au Paléolithique selon que l'animal est vivant - coquillage -, ou mort - squelette, coquille -, nous conduit tout naturellement à apprécier la notion de distance. Les contraintes du transport d'un animal utilisé frais ne sont pas les mêmes que celles du test coquillier. De même le message ou la symbolique qu'ils véhiculent peuvent être très différents. Mais, ne suffit-il pas d'extraire l'animal de son abri pour faire du coquillage une coquille? C'est une notion que nous pouvons aborder dès le Mésolithique le long du littoral atlantique français jusqu'à l'Antiquité. 
L'analyse du coquillage en tant que ressource alimentaire demeure un exercice nouveau en archéologie. Mais suffit-il à l'Homme d'avoir accès à des espèces comestibles pour les consommer? Si certaines contraintes liées au transport peuvent être évoquées comme la nécessité de garder les coquillages au frais ou de les transformer pour qu'ils restent comestibles, d'autres facteurs semblent intervenir dans le filtre de la consommation alimentaire, de la Préhistoire à nos jours.

En outre, le développement de techniques de fouille adaptées aux invertébrés marins et l'usage de plus en plus systématique d'un tamisage à maille fine (2 $\mathrm{mm}$ ) autorisent la découverte d'espèces de petites dimensions, par le passé, invisibles en archéologie. Si ces mollusques ont bel et bien été transportés par l'homme sur leur lieu d'habitat, ils l'ont été bien souvent involontairement. Ils restent malgré tout les témoins d'activités anthropiques.

\section{Dis-moi quels coquillages tu nás pas mangé, je te dirai quelles coquilles tu as utilisées}

\section{Diversité des activités liées aux mollusques marins et découvertes en archéologie}

L'analyse archéologique de l'ensemble des restes coquilliers découverts le long du littoral atlantique français permet de mettre en évidence différentes utilisations des coquillages. Au cours de la Préhistoire, l'alimentation, la parure et l'outil sont les principales recensées (Gruet 1993; Dupont 2006) ; les deux premières sont encore d'actualité bien que les critères de leur sélection aient évolué. Parmi les parures les plus récentes découvertes en archéologie, la coquille Saint-Jacques, attribut du pèlerin, est régulièrement mise au jour dans les sépultures à partir du $x^{e}$ siècle (Gruet et Bonnissent 2002). La Protohistoire procure de nombreux exemples de comblement des structures d'habitats grâce aux coquilles logées dans les interstices des murs de pierres. L'habitat du Bronze ancien de Beg-ar-Loued localisé sur l'archipel de Molène (Finistère) en témoigne. On relève encore d'autres usages sous l'Antiquité tels que les pavages d'huîtres mis en évidence par la fouille de l'École des beaux-arts de Nantes dirigée par Nicolas Rouzeau. Il existe également des épandages plus anarchiques qui servent à drainer les sols ou les voies de circulation comme sur le site de la Pouplinière (Saint-Michel-Chef-Chef, Loire-Atlantique, responsable d'opération D. Doyen). À partir du $\mathrm{ul}^{\mathrm{e}}$ siècle ap. J.-C., une mode venue d'Italie semble influencer l'ornementation des villas côtières de l'Armorique (Boislève et al. 2011). La surface intérieure des murs de plusieurs dizaines de villas est incrustée de milliers de coquilles. Dans des sites plus récents, les coquilles présentent encore des originalités. Ainsi ces quelques exemplaires exotiques, témoins de voyages lointains effectués au xvII ${ }^{e}$ siècle, découverts sur des chantiers archéologiques de Charente-Maritime. Enfin, l'une des utilisations 
oubliées du patrimoine côtier atlantique, la confection de teinture à partir du pourpre Nucella lapillus et du murex Ocenebra erinaceus, mérite également d'être rappelée (Gruet 1993; Dupont 2011).

Si la destination alimentaire ou tinctoriale des coquillages nécessite que ceux-ci soient collectés avec leur chair, tel n'est pas le cas pour les autres utilisations où la coquille est exploitée en tant que matière première. À une époque, où les préoccupations écologiques sont de mises, poser la question du possible recyclage des coquilles, une fois l'animal extirpé de son habitacle, ne nous paraît pas infondé. L'archéologie permet d'y répondre, aux moyens de plusieurs analyses. C'est ainsi que les spectres malacofauniques des espèces consommées, c'est-à-dire, de celles trouvées dans les zones de dépotoirs archéologiques, peuvent être confrontés aux spectres d'espèces liées à d'autres utilisations.

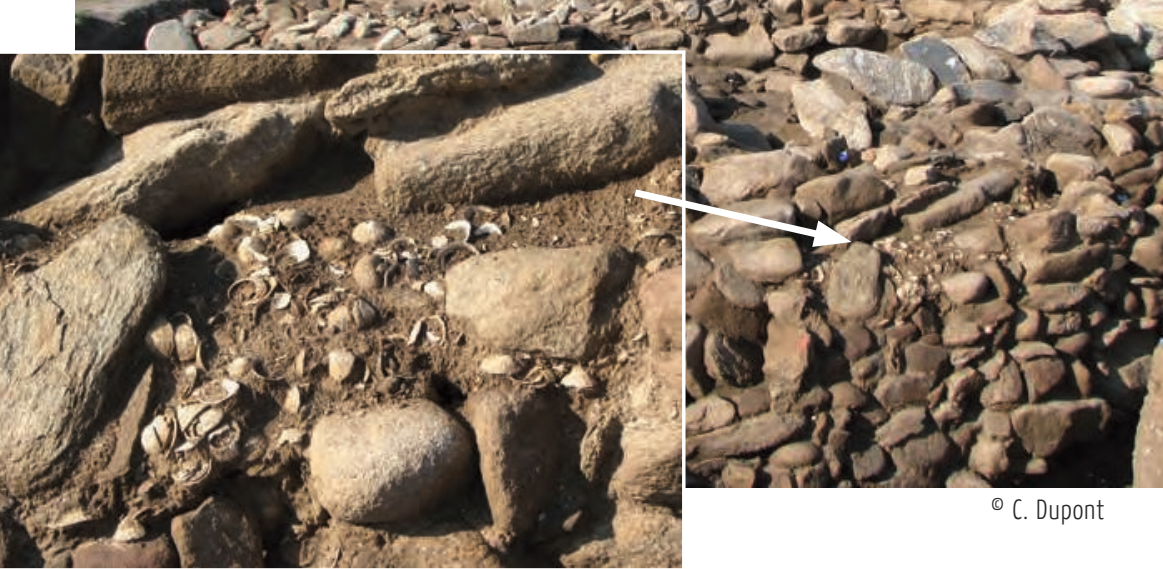

Les patelles, ressource alimentaire majeure le long des côtes rocheuses ont été recyclées pour combler le système de double parement observé à Beg-ar-Loued à l'âge du bronze (île de Molène, Finistère). (Fig. 1)

\section{De l'objet utilitaire au symbole}

La comparaison entre les espèces mangées et celles dont la coquille seule est utilisée nous amène à présenter deux statuts différents de mollusques marins: d'une part les mollusques dont les tests ne sont qu'utilitaires, de l'autre ceux semblant davantage chargés de symbolique.

Dans le premier cas, des déchets alimentaires paraissent être utilisés, dans le second l'acquisition même de la matière a l'air de correspondre à une activité bien distincte. Le recyclage des déchets comble les vides (interstices des murs, ornières, (Figure 1)) et sert à se débarrasser d'une accumulation encombrante de coquille. Ce type d'utilisation reste cantonné au littoral et passe pour purement utilitaire.

Dans le second cas, les espèces de mollusques consommés sont très minoritairement représentées parmi les coquilles utilisées. La figure 2 illustre simultanément les espèces majoritairement consommées au Mésolithique final, le long du littoral atlantique français, et celles qui, percées, portent les stigmates d'usure attestant de leur utilisation comme parure. Divers critères de distinction justifient de classer séparément les parures et les espèces consommées. D’une façon générale, la taille des coquillages collectés pour la consommation est supérieure à deux centimètres, ce qui n'est pas le cas pour la plupart des espèces utilisées comme parure au Mésolithique (Dupont 2006). Il semble que les hommes du Mésolithique préfèrent, pour se sustenter, pratiquer la pêche à pied à marée basse plutôt que l'immersion même dans des eaux peu profondes. Ils attendent que la marée baisse et que l'estran ou zone intertidale se découvre pour collecter leurs repas. Certaines parures, quant à elles, sont, comme le dentale, représentées par des espèces subtidales qui vivent sous le niveau des plus basses mers (Poppe et Goto 1993). Elles 
À gauche, les espèces majoritairement consommées au Mésolithique le long du littoral atlantique français ; à droite, celles qui sont majoritairement utilisées en tant que parure ; 1 : I'huître plate Ostrea edulis, 2 : la patelle Patella sp.

3 : la scrobiculaire Scrobicularia plana,

4 : la coque Cerastoderma edule, 5 : la moule Mytilus edulis, 6 : la littorine obtuse Littorina obtusata,

7 : la cyprée Trivia monacha, 8 : le dentale Dentalium sp., 9 : la nasse réticulée Nassarius reticulatus (coquilles de Beg-an-Dorchenn, Plomeur, Finistère)

(Fig. 2)
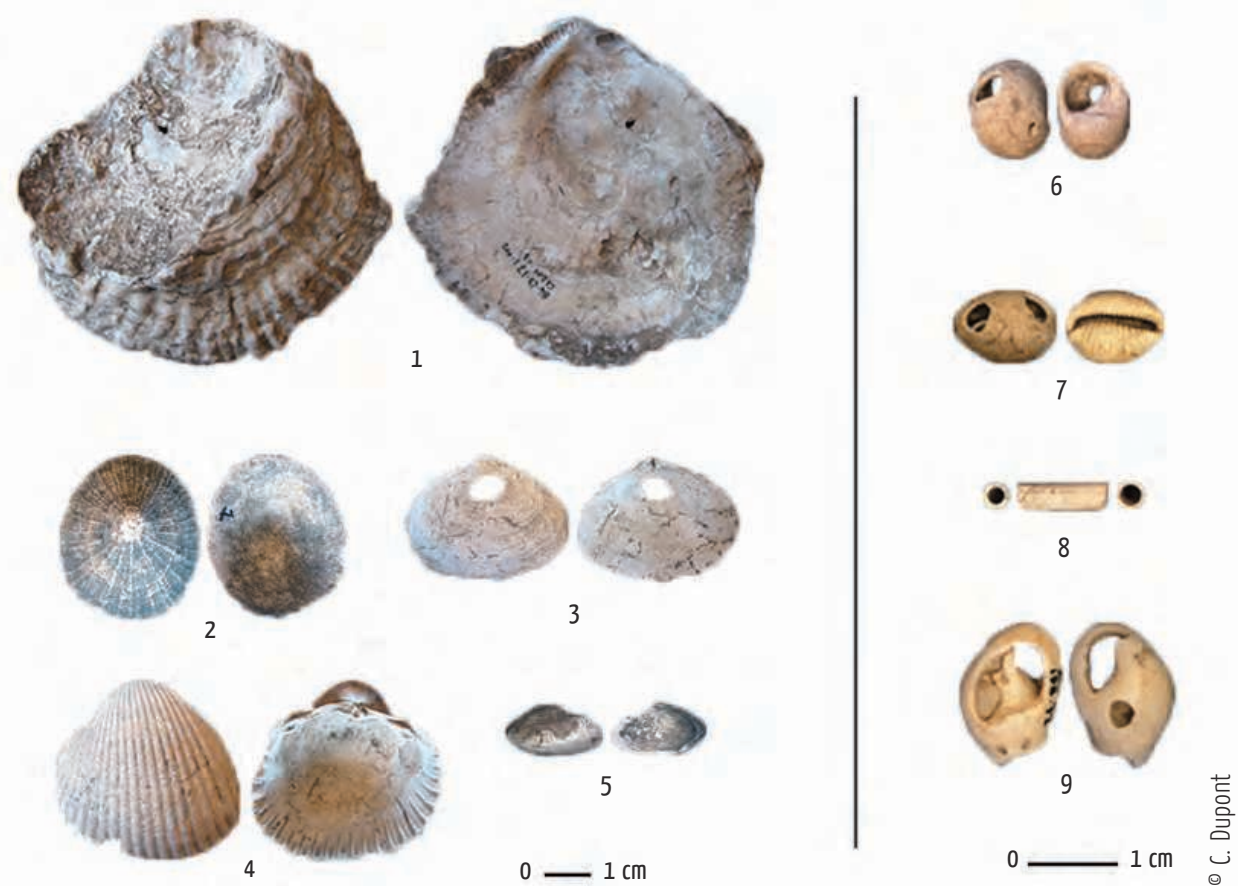

n'ont cependant pas été collectées vivantes. Leurs états de surface présentent parfois des polis et cassures liés au ressac de la mer. Ces traces montrent que les coquilles utilisées comme parure ont été ramassées alors qu'elles étaient échouées et déjà dépourvues de la chair de l'animal. La nasse réticulée de l'amas coquillier de Beg-an-Dorchenn (Figure 2 $\left.n^{\circ} 9\right)$ en présente un très bon exemple. Il est d'ailleurs fort probable que l'érosion marine soit à l'origine des deux perforations présentes sur cet exemplaire. Si des patelles et des coques ont pu être décrites en tant que parure sur le territoire breton (Giot et al. 1998), l'analyse de leur état de surface montre que beaucoup de ces perforations sont dues à des processus taphonomiques et aucun stigmate n'atteste de leur utilisation en tant que parure (Dupont et al. 2010). Ainsi, ce n'est nullement la valeur alimentaire des coquilles qui leur confèrent le statut de parure. Le cas des coquilles Saint-Jacques, témoins du pèlerinage de Saint-Jacques-de-Compostelle est tout à fait intéressant. Ces tests semblent pour certains avoir été taillés, modifiant leur aspect général. Au XII ${ }^{e}$ siècle des enseignes métalliques viendront copier la forme de ces coquilles taillées, s'éloignant de la forme originelle de la coquille Saint-Jacques (Gruet, Bonnissent 2002). Ce n'est pas la coquille qui semble nécessaire mais ce qu'elle représente. Un autre critère, souvent absent des échantillons archéologiques, a aussi pu participer au choix de cette coquille: sa couleur.

En archéologie, cette valeur est perceptible grâce aux coquilles utilisées dans les décors muraux des villas du III $^{e}$ siècle ap. J.-C. (Boislève et al. 2012). Les tests sont apposés sur des plages colorées et différentes espèces sélectionnées selon les couleurs de ces plages (Figure 3). De nombreuses similitudes rapprochent des parures préhistoriques, les coquilles antiques qui ornent les décors muraux. Si plusieurs des espèces utilisées incrustées sont comestibles et consommées dans le Sud de la France pendant l'Antiquité, en Armorique au III s. ap. J.-C. (Boislève id.), ce ne fut pas le cas. La coutume perdure actuellement. Tandis que sur la côte méditerranéenne on se délecte des donaces maladroitement appelées tellines, les 
mêmes coquillages sont boudés sur les côtes bretonnes. Ces coquilles, à l'instar des parures préhistoriques, ne sont pas issues du recyclage de coquillages consommés. À nouveau, la présence de perforations naturelles et l'aspect roulé des coquilles témoignent de leur collecte à l'état d'échouage et dépourvues de la chair de l'animal (Figures 3 et 4). Comme pour la parure, des tests inférieurs à $2 \mathrm{~cm}$ (Littorina obtusata, Gibbula sp.) et des espèces subtidales ont été répertoriés parmi ces assemblages; par exemple, l'Acanthocardia tuberculata (Figure 4) souvent confondue en archéologie avec la coque commune Cerastoderma edule. De tels

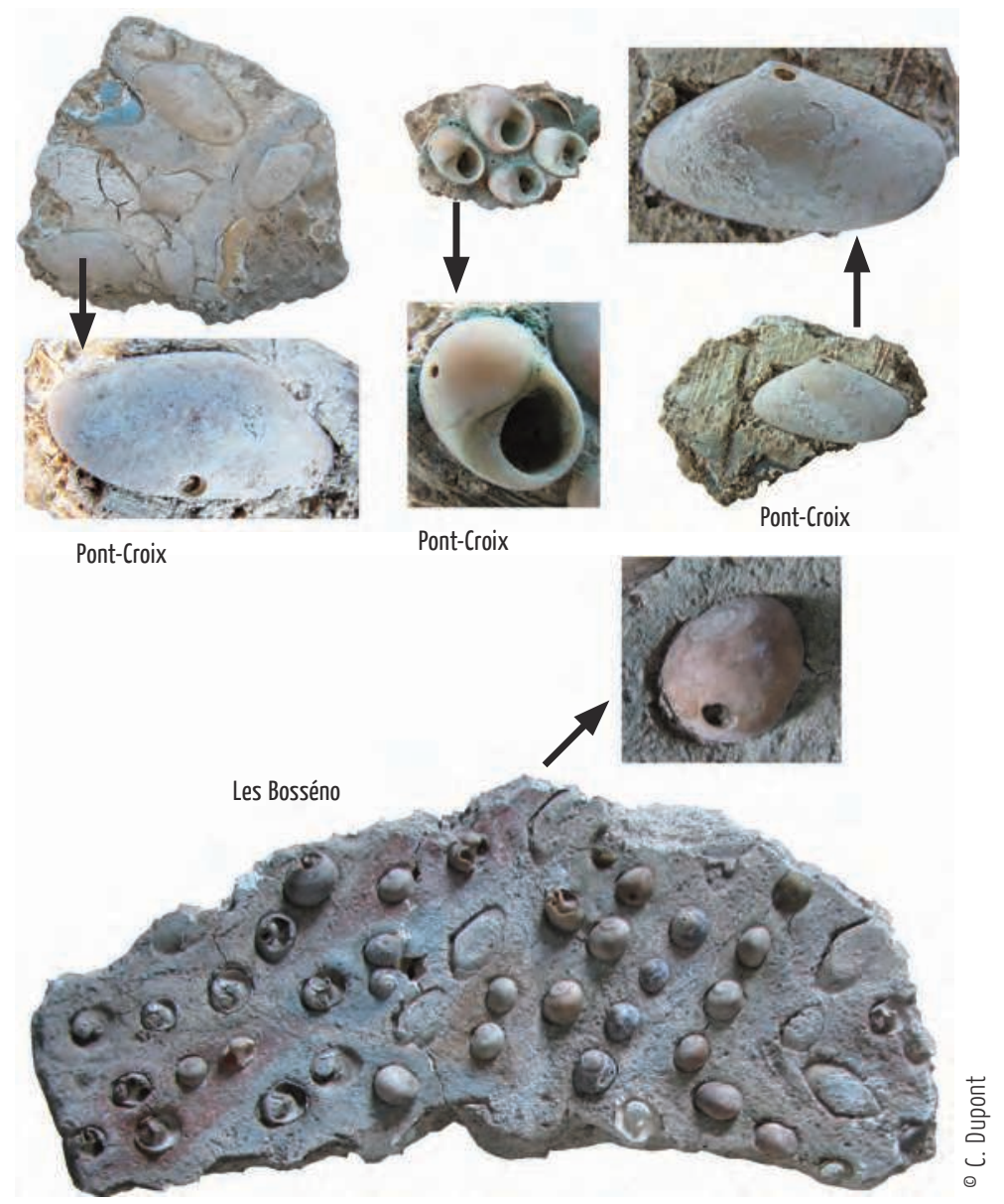

Certaines coquilles sont percées naturellement. Ces stigmates indiquent que ces coquillages étaient déjà morts quand leurs tests ont été ramassés.

À noter en bas :

le fragment de décor allie différentes espèces mollusques aux différentes plages colorées.

(Fig. 3)

décors donnent le sentiment d'une recherche de rendu général plutôt que d'une sélection de gabarits parfaitement maîtrisés. Certaines des coquilles étaient déjà cassées lorsqu’elles ont été incorporées dans les décors, d'autres l'ont été lors de leur application (Figure 5). Un caillou roulé de quartz est même venu imiter unes des nombreuses littorines obtuses de la villa Les Bosséno (Figure 3). L'ensemble de ces éléments témoigne davantage d'un choix stylistique que d'un choix strictement lié à l'accessibilité de la ressource. Le rappel du milieu aquatique invoque autant « le contexte mythologique de certains épisodes [...] que le lieu de vie des nymphes » (Boislève id.). L'influence marine des décors muraux de ces villas gallo-romaines peut être observée à plus de soixante kilomètres du trait de côte. 


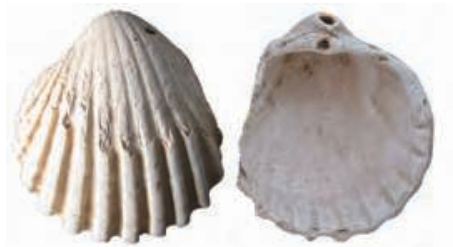

1
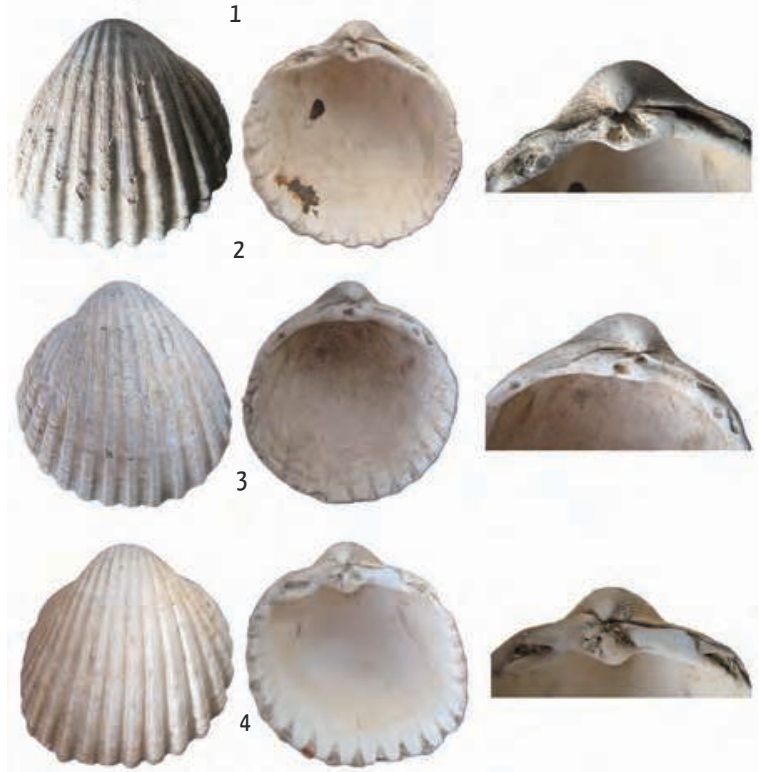

Stigmates d'usure marine observés sur les Acanthocardia tuberculata, des plus prononcés $n^{\circ} 1$, aux plus faibles $n^{\circ} 4$ (villa de Mané Véchen, Plouhinec, Morbihan).

$1: 62 \mathrm{~mm} ; 2: 59 \mathrm{~mm} ; 3: 51 \mathrm{~mm} ; 4: 58 \mathrm{~mm})$.

(Fig. 4)

Certaines coquilles inclues dans les décors étaient déjà cassées lorsqu'elles ont été appliquées sur leur support.

(Fig. 5)
Strombus pugilis juvénil au test usé par la mer (La Maison Champlain, Brouage, Charente-Maritime.

(Fig. 6)

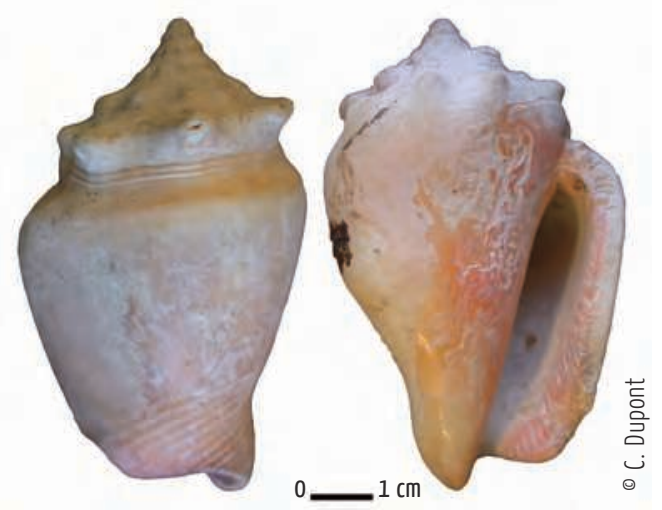

On peut également évoquer ici, les rocaillages plus tardifs des labyrinthes du château de Versailles essentiellement composés de coquilles exotiques. De rares exemplaires d'espèces exotiques sont découverts sur des sites archéologiques d'époque moderne. Leur recensement manque mais nous pouvons citer les strombes Strombus pugilis des sites de la Maison Champlain à Brouage et du Théâtre Verdière à La Rochelle, tous deux localisés en CharenteMaritime et découverts dans des accumulations datées du XvII ${ }^{\mathrm{e}} \mathrm{s}$. (Figure 6). Si cette espèce a pu être consommée, ici, c'est le souvenir de contrées lointaines que la coquille semble véhiculer. L'exemplaire de Brouage présente un aspect roulé qui témoigne de sa collecte alors qu'il était déjà mort.

La collecte de coquilles échouées sur la plage peut avoir une autre finalité: la recherche d'outils (Gruet 1993). Le statut de ces tests est différent des parures ou des décors muraux. Leur sélection résulte plus de contraintes techniques que d'une éventuelle symbolique, et le recyclage de certaines valves de mollusques ne peut être exclu (Dupont 2006). Cette forme d'utilisation, sur le littoral atlantique français, est principalement connue au Mésolithique et au Néolithique (Figure 7). Au Mésolithique les tests utilisés comme outils semblent rejetés dans la masse des déchets culinaires et participer à des activités expéditives, tandis qu'au Néolithique, les sites qui présentent une telle utilisation sont parfois distants de la côte

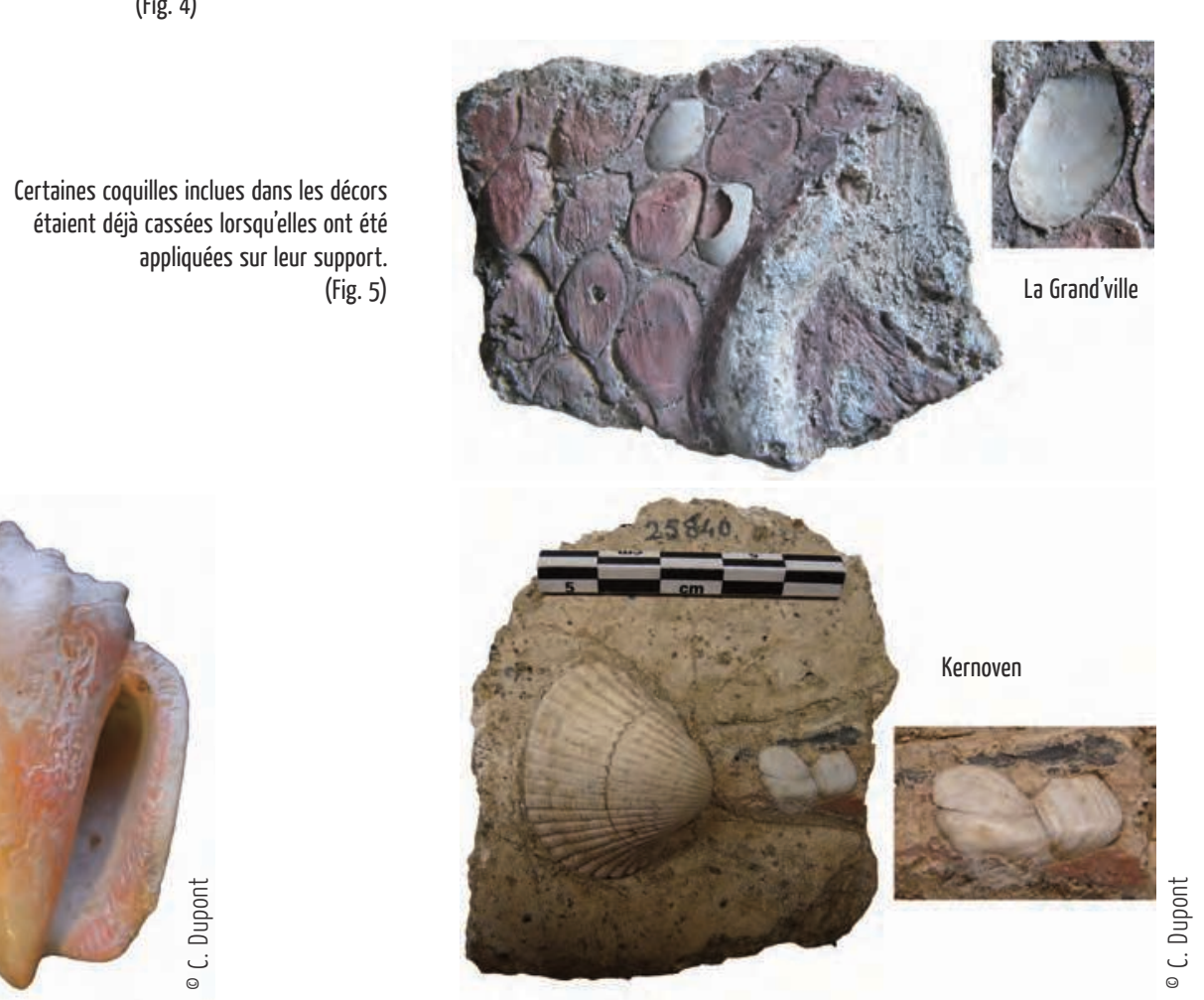



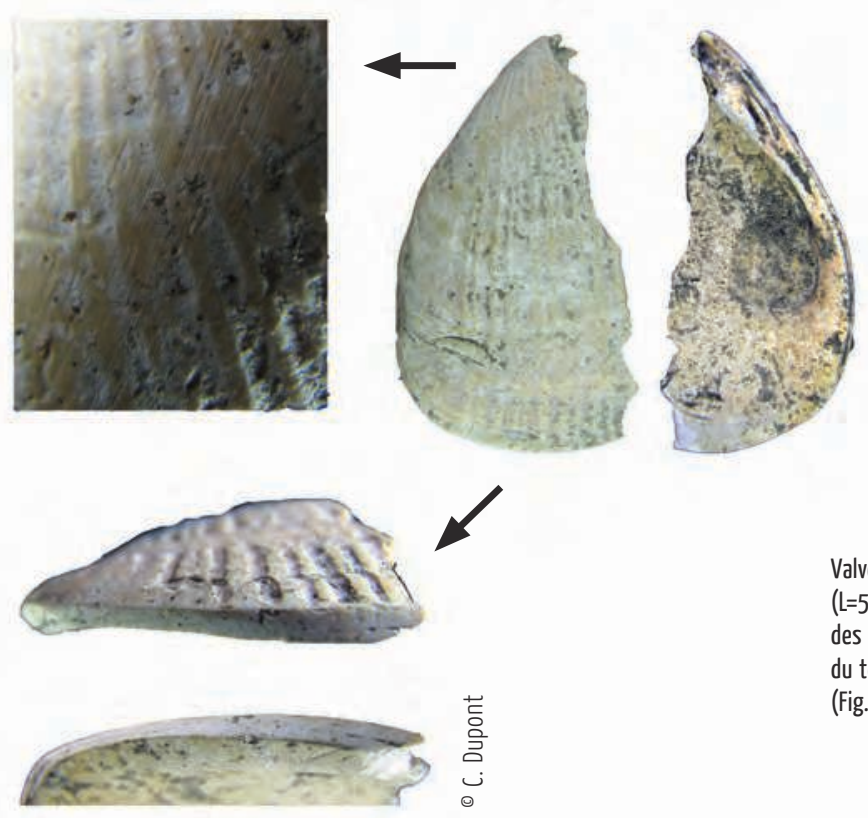

Valve de Laevicardium crassum utilisée comme outil ( $(=54 \mathrm{~mm})$ au Néolithique en Charente-Maritime avec des stries d'abrasion sur la surface externe du test et du bord du test (Fig. 7)

de plus de trente kilomètres (Gruet et al. 1999; Dupont 2006). Une autre différence, au Néolithique, est leur possible stockage dans des fosses.

La coquille est donc un artefact qui circule et véhicule le plus souvent un imaginaire foisonnant, à partir d'un univers marin difficilement maîtrisable. Les coquillages collectés frais nous apportent quant à eux d'autres informations sur la gestion du territoire, dès la Préhistoire.

\section{Gestion du territoire et déplacements liés à l'exploitation des coquillages frais}

Cette notion de prestige qui s'attache à la gestion du territoire peut également être associée à des coquillages consommés. Au fil de la chronologie, l'apparition des filtres commerciaux permet, avec le développement des voies commerciales et des moyens de transport, de mettre en évidence les coquillages les plus appréciés des populations les plus aisées qui vivent à plusieurs centaines de kilomètres du trait de côte. Cette distinction entre ressources marines consommées près de la côte et à l'intérieur des terres n'a pas toujours été possible.

\section{Du chasseur-cueilleur au producteur}

En effet, la synthèse des sites archéologiques qui témoigne de la consommation de mollusques marins au Mésolithique montre, à l'échelle de la façade atlantique européenne, que cette consommation ne dépassait pas la distance d'un kilomètre par rapport aux influences marines (Figure 8). Ainsi, certains sites qui apparaissent actuellement plus 


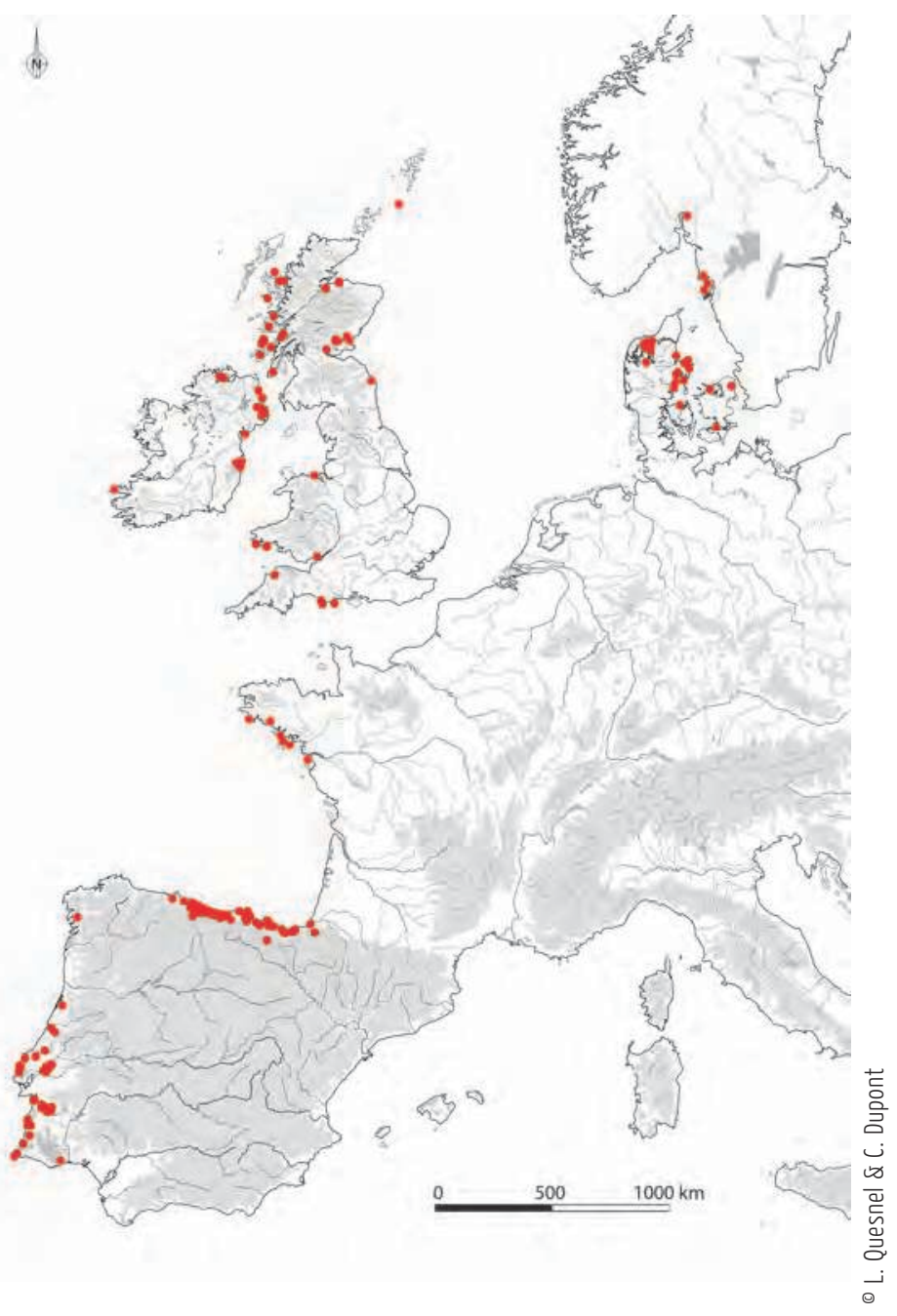

Distribution des amas et dépotoirs coquillers du Mésolithique le long du littoral atlantique européen.

(Fig. 8) éloignés de la mer sont des habitats positionnés au Mésolithique près de larges estuaires. Ils subissaient pour la plupart l'influence de la salinité au pied des campements (exemples au Portugal des estuaires du Tage et du Sado). Cette exploitation de ressources marines accessibles quotidiennement à marée basse n'a rien d'anodin. Elle semble avoir eu un impact majeur dans l'évolution de nos populations et a peut-être encouragé les derniers chasseurs-cueilleurs du littoral atlantique européen à établir une certaine permanence des habitats côtiers (Hodder 1993; Schulting 1996; Dupont 2006). Lanalyse croisée des ressources marines et de l'industrie lithique a permis de proposer deux types de sites côtiers avec exploitation des coquillages (Figure 9, Dupont \& Marchand 2008). Le premier type de site semble correspondre à des stations logistiques littorales avec un temps de résidence plus éphémère. Cette hypothèse est basée sur la restriction de l'exploitation des ressources marines aux seuls coquillages et sur un nombre d'espèces très réduits. Le second type de site est le camp de base pour lequel les ressources marines exploitées sont des plus diversifiées. Cette diversité est lisible à la fois au niveau des taxons représentés mais aussi au niveau des classes animales. En effet, crabes, poissons, oiseaux marins accompagnent les coquillages dans les menus des hommes du Mésolithique. La présence de structures d'habitat lourdes, de nécropoles, d'outils en silex associés à différentes activités participe à la notion d'une certaine permanence de ces habitats. L'analyse de la saison de collecte des mollusques marins et les calendriers d'accessibilité facilitée des ressources montrent que ces populations ont pu s'établir sur la frange côtière pendant plusieurs mois (Dupont et al. 2009). Ainsi, l'abondance et la diversité des ressources marines dont les coquillages ont pu faciliter le passage d'un mode de résidence nomade à sédentaire. Qu'en est-il au Néolithique avec l'arrivée de l'agriculture? Si la sédentarisation se poursuit, les populations néolithiques semblent littéralement tourner le dos aux ressources marines et aux coquillages. Les dépotoirs coquilliers découverts en archéologie sont moins volumineux (Dupont 2006) et les analyses isotopiques réalisées sur les squelettes humains montrent que les ressources marines entrent très secondairement dans le régime alimentaire (Schulting et al. 2004; Schulting 2005). Est-ce l'arrivée de l'agriculture qui est à l'origine de ce phénomène avec la présence de ressources alimentaires plus prévisibles? Les coquillages passent-ils alors du statut de ressource alimentaire nécessaire à la survie des populations côtières à celui de ressource secondaire? Les espèces consommées au Mésolithique (les patelles Patella spp., l'huître plate Ostrea edulis, la moule Mytilus edulis, la coque Cerastoderma edule, la scrobiculaire Scrobicularia plana) le sont aussi au Néolithique et l'analyse des vestiges archéologiques montre que l'accessibilité reste le principal facteur qui a influencé leur sélection (Dupont 2006). 


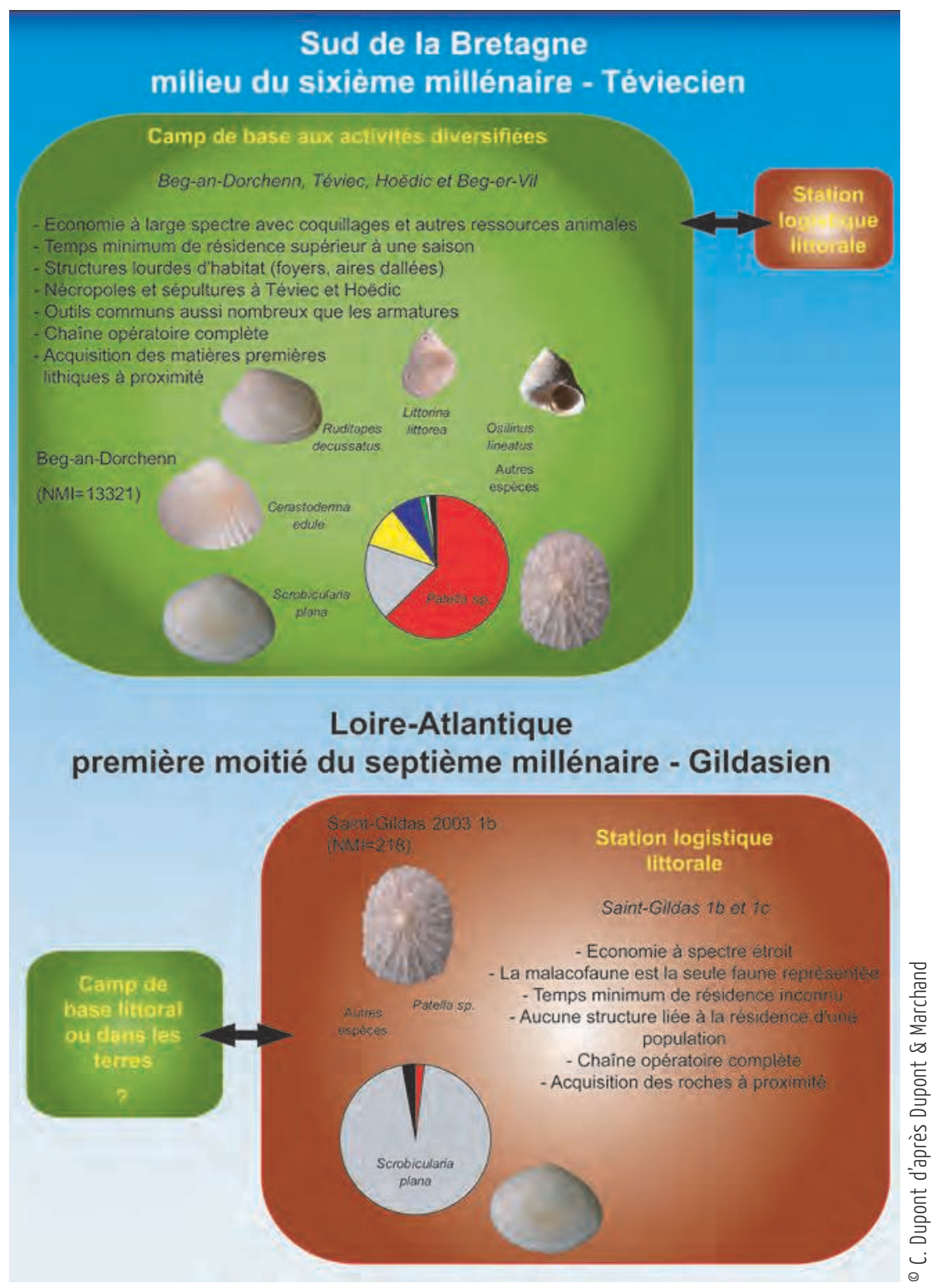

Système d'organisation logistique de la bande côtière à la fin du Mésolithique le long du littoral atlantique français

(NMI : Nombre Minimum d'Individus). (Fig. 9)

\section{Le filtre culturel}

Si les proportions entre les espèces consommées peuvent varier au fil de la chronologie, ce sont les mêmes taxons qui composent les régimes alimentaires des hommes de façon récurrente de la Préhistoire à l'époque moderne à proximité des côtes atlantiques et de la Manche en France (Figure 10). Laccélération des transports dès l'Antiquité voit peu à peu la consommation des coquillages se développer loin du trait de côte. La moule Mytilus edulis, la coque Cerastoderma edule et la palourde Ruditapes decussatus accompagnent régulièrement l'huître plate Ostrea edulis par le biais de voies commerciales (Dupont \& Blondiau 2006). La consommation de la patelle Patella spp., la monodonte Osilinus lineatus, le bigorneau 

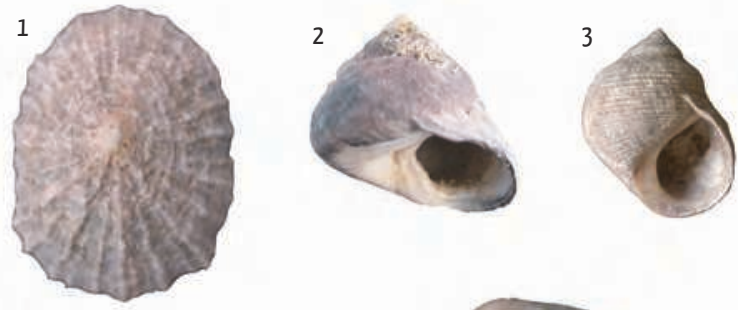

4
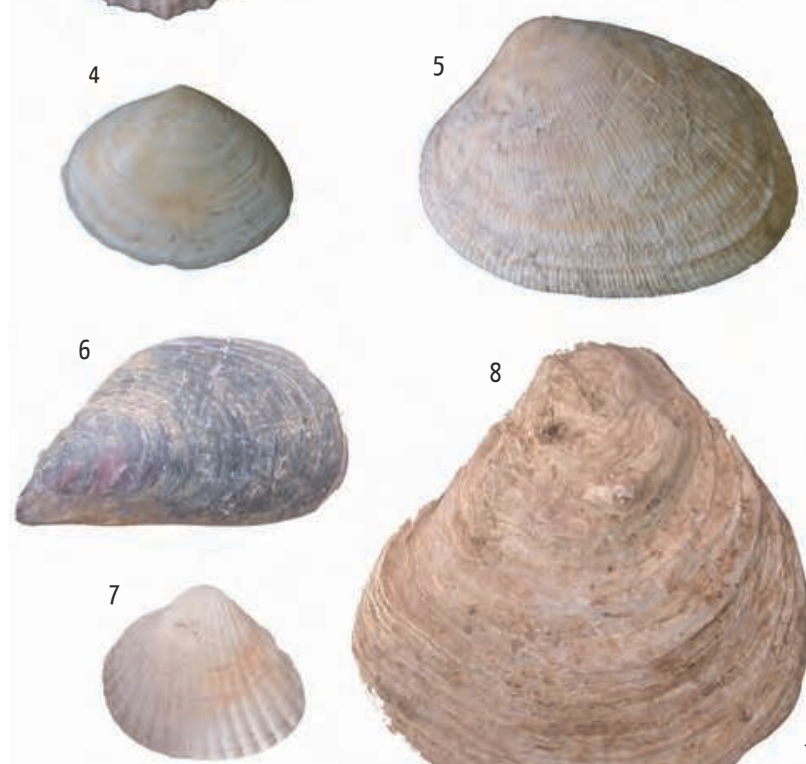

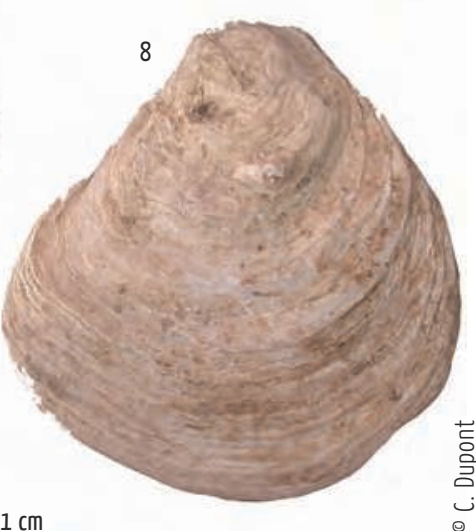

$1 \mathrm{~cm}$

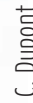

Mollusques marins les plus abondants dans les dépotoirs archéologiques du littoral atlantique français de la Préhistoire à l'époque moderne

1 : la patelle Patella spp., 2 : la monodonte Osilinus lineatus, 3 : le bigorneau Littorina littorea, 4 : la scrobiculaire Scrobicularia plana, 5 : la palourde européenne Ruditapes decussatus, 6 : la moule Mytilus edulis, 7 : la coque Cerastoderma edule, 8 : l'huître plate Ostrea edulis (Fig. 10)

Parmi les zones dépotoir de La Plaine-Tatihou, ce refus de tamis daté des $x \|^{e}{ }^{e}$ et xive siècles témoigne

de la consommation de patelles Patella spp., de bigorneaux Littorina littorea, d'huîtres Ostrea edulis et de monodonte Osilinus lineatus (SaintVaast-La-Hougue, Manche)

(Fig. 11)
Littorina littorea et la scrobiculaire Scrobicularia plana semble quant à elle se cantonner près de la côte (Figure 11). Dès le développement des voies de circulation, la notion de transport induit donc un filtre culturel et ce, même pour les coquillages alimentaires.

Comparer ces espèces phares à ce que nous trouvons actuellement dans nos assiettes est intéressant. Le facteur de conservation n'est plus un facteur limitant dans le choix des espèces que nous consommons, contrairement aux époques antérieures. Si les patelles et les scrobiculaires sont mangées dans d'autres pays européens comme le Portugal, ces deux variétés ne sont quasiment plus consommées le long du littoral atlantique français. La poignée de personnes qui les consomme aujourd'hui vit à quelques kilomètres de la mer et ne représente qu'un épiphénomène. Les périodes de disette (Wickham-Jones 2003) et la seconde guerre mondiale sont les causes en partie liées de cette désaffection. Les populations proches des côtes ont été durant le conflit obligées de manger fréquemment ces deux coquillages, les associant à une image négative. Ce type de nourriture tout comme le topinambour a été retiré de nos menus après guerre. Le cas de la monodonte Osilinus lineatus semble différent. Le gastéropode est régulièrement découvert dans la plupart des zones dépotoirs des sites archéologiques côtiers. Cette espèce est cependant absente de nos plateaux de fruits de mer actuels. Une des raisons qui pourrait expliquer le fait qu'elle soit boudée se trouve peut-être associée, à l'origine, à un facteur environnemental. En effet, cette espèce est particulièrement sensible aux hivers très froids. Ainsi, lors des hivers des années 1962-1963, elle a quasiment disparu de nos estrans; elle n'est revenue les coloniser que vingt ans plus tard (Mieszkowska et al. 2006). Est-ce sa disparition transitoire qui a fait disparaître ce gastéropode de nos assiettes? Il existe, à l'inverse, des espèces actuellement consommées qui

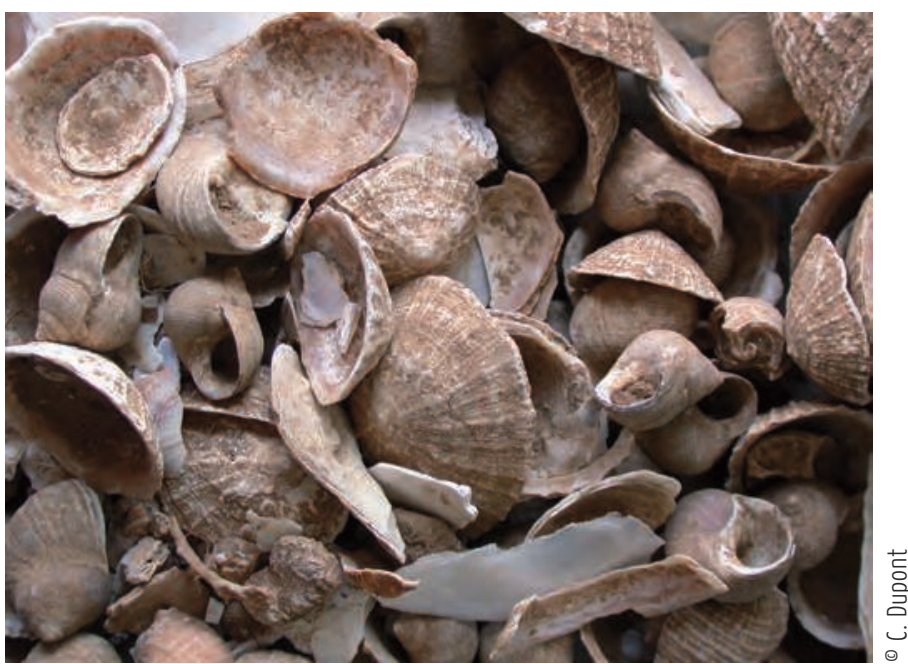


ne sont pas trouvées dans les rejets alimentaires archéologiques; c'est le cas de l'amande de mer Glycymeris glycymeris, du buccin Buccinum undatum et de la coquille Saint-Jacques Pecten maximus. Ces espèces sont très abondantes dans la zone subtidale (Poppe et Goto, 1991 ; 1993) et il est fort probable que le développement des techniques de dragage soit à l'origine de leur récente consommation.

\section{Le colorant pourpre}

Lutilisation du coquillage en tant que nourriture n'a pas l'exclusivité de la nécessaire fraîcheur ni de la transformation juste après sa collecte. Il existe le long du littoral atlantique français des sites archéologiques qui témoignent de l'extraction de colorant à partir de la glande tinctoriale du pourpre Nucella lapillus et du murex Ocenebra erinaceus (Cardon 2003). Cette activité a été mise en évidence depuis la Tène finale jusqu'au Moyen-Âge ( IIV $^{e}$ s.) le long de la Manche et de la côte atlantique française (Figure 12; Dupont 2011). La réaction chimique qui permet la coloration nécessite l'extraction de la glande hypobranchiale tandis que le mollusque est encore frais (Cardon 1990). Cette caractéristique se traduit par une distribution géographique des sites archéologiques en contact direct avec la mer (Figure 12). Cette distance d'exploitation du pourpre impose un contrôle de la matière colorante le long de la frange côtière et a pu accentuer le prestige véhiculé par la couleur obtenue. À cette restriction géographique s'ajoute la nécessité de briser latéralement (Figure 13) ou d'écraser (Figure 14) des milliers de coquillages. Les quantités nécessaires montrent à quel point ces coquillages ont eu une haute valeur ajoutée.

Il existe également dans le registre archéologique, d'autres coquillages qui peuvent rester invisibles si les techniques de fouille ne sont pas adaptées. La multiplication des prélèvements sédimentaires associée à un tamisage fin $(2 \mathrm{~mm})$ a permis de mettre en

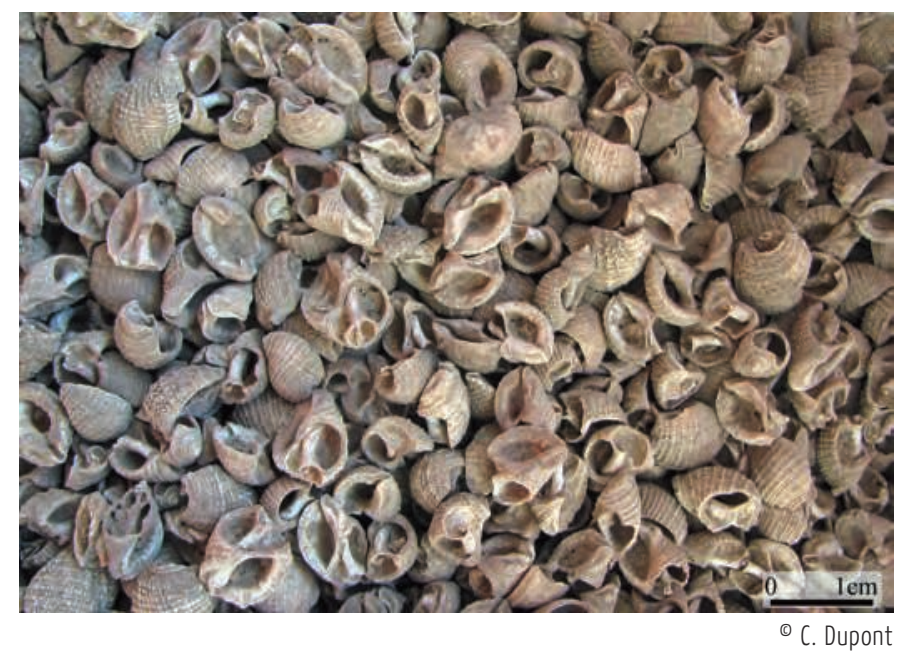

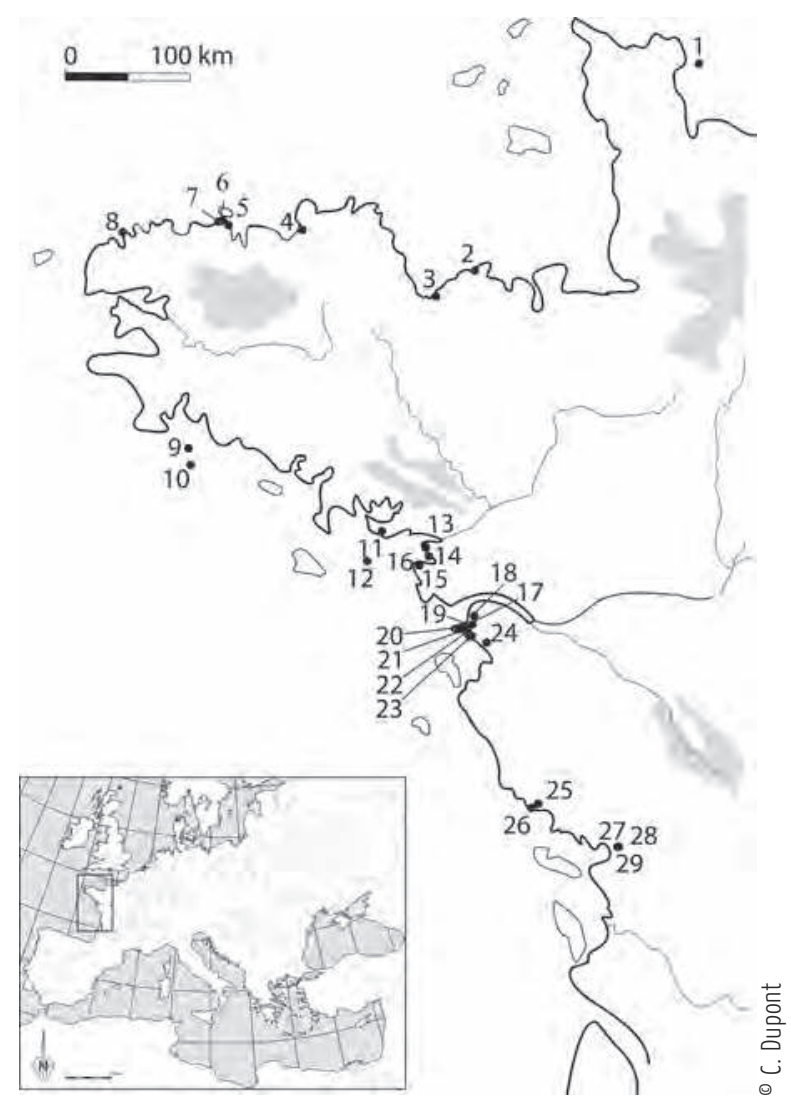

Localisation des sites archéologiques français associés à des pourpres Nucella lapillus utilisés à des fins tinctoriales

1- La Plaine, 2- Sables-d'Or, 3- Granville, 4- Le Yaudet, 5- Pointe du Béron, 6- Prat, 7- Staol, 8- Tariec Vraz, 9- Sondages 1 et 2, île aux Moutons, 10- Île de Brunec, 11- Tréutan, 12- Port-Blanc, 13- Plage du Lomer, 14- Pen-Bé, 15- ZA du Pladreau, 16- Le Clos du Moulin, 17- La Cossonière-des-Rives, 18- La Pouplinière, 19- La Lucette, 20- Moulin Tillac, 21- Comtée, 22- La Cornillais, 23- ZAC des "Terres aux Moines", 24- Les Missotières, 25- Le Grand Essart, 26- La Payré 2 , 27- Champ du Bois, 28- Bel Air, 29- les Plantes (Fig. 12)

Coquilles de pourpres Nucella lapillus cassées latéralement associées au niveau de la fin de l'Antiquité au Moyen-Age sur le site archéologique du Grand-Essart (Jard-sur-Mer, Vendée).

(Fig. 13) 


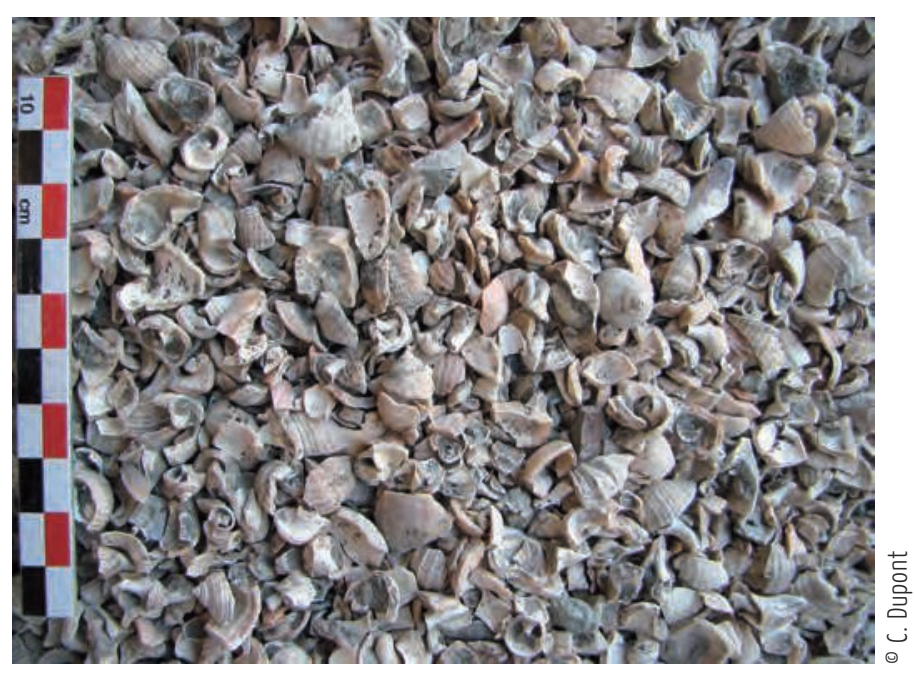

Coquilles de pourpres Nucella lapillus concassées associées au niveau antique du site archéologique de La Pouplinière (Saint-Michel-Chef-Chef, Loire-Atlantique) (Fig. 14)

L'épandage d'algues pour amender les terres est encore d'actualité dans les potagers bretons (Fig. 15) côtières. lumière d'autres mollusques qui restent liés à la notion de transport mais dont la volonté humaine n'a pas été de les collecter un à un sur l'estran ou la grève.

\section{Témoins archéologiques de mollusques marins transportés malgré eux}

L'exploitation de certaines ressources liées à la mer a pu induire le transport de coquillages marins souvent de petite dimension (inférieure à $1 \mathrm{~cm}$ ). Le corpus archéologique permet de les découvrir à plus ou moins longues distances du trait de côte.

Les algues marines ont été le vecteur du transport de gastéropodes marins. En effet, elles sont transportées pour garder au frais certains aliments, elles peuvent être séchées pour servir de combustible ou pour faire de la soude, elles peuvent également être consommées et servir à amender les terres (Beaune 1995; Gruet \& Dupont 2009; Pailler et al. 2011, Figure 15). Ce sont alors les coquillages marins vivants accrochés aux algues qui sont transportés vers les lieux de vie des populations

Les sables coquilliers marins ont aussi été ramassés pour entrer dans la composition de mortier pour la construction de bâtiment par exemple. Contrairement au vecteur précédent, le sable coquillier correspond à des coquilles dépourvues de la chair de l'animal. De nombreux stigmates témoignent de cet état du test coquillier (Figure 16). Certaines parties de la coquille manquent ou sont roulées. Le transport d'argile pour confectionner

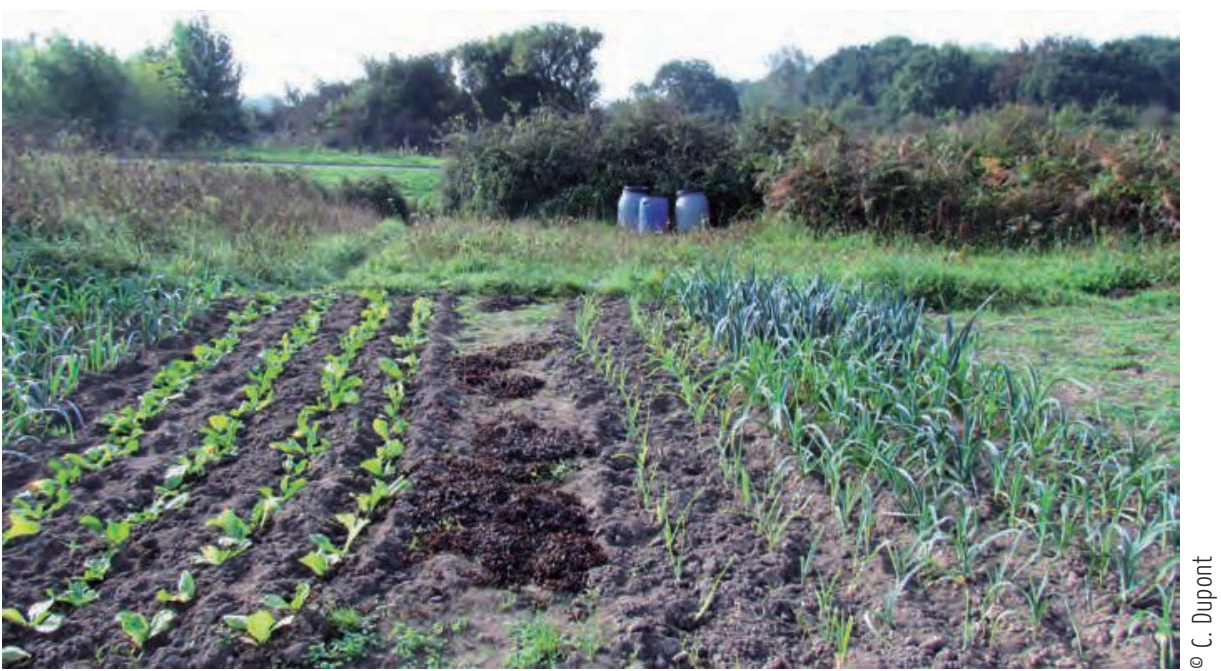




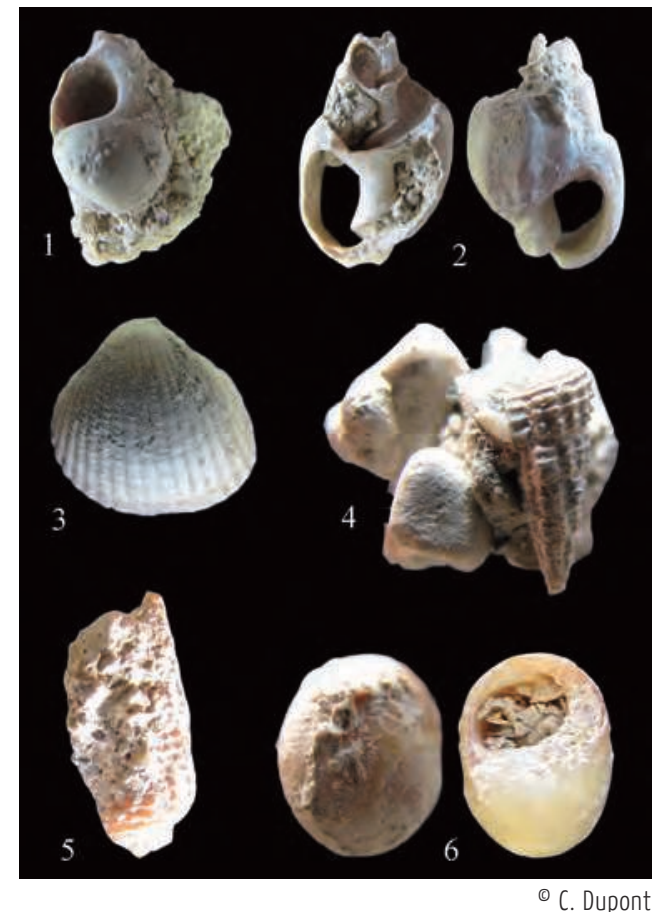

${ }^{\circ}$ C. Dupont

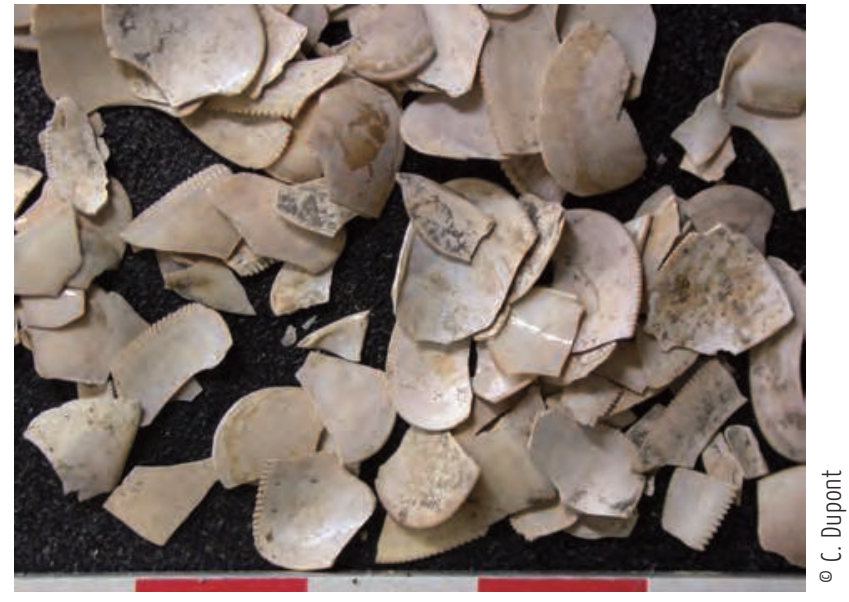

Ces fragments de bivalves ont été découverts dans les zones dépotoirs du château médiéval de Boves (Sommes).

(Fig. 17)

sata $(\mathrm{l}=21$ et $11 \mathrm{~mm}) ; 2$ : Nassarius reticulatus

$(\mathrm{L}=20 \mathrm{~mm}) ; 3$ : Cerastoderma edule $(\mathrm{L}=26 \mathrm{~mm})$;

4 et 5 : Bittium reticulatum ( $(=9$ et $8 \mathrm{~mm})$

(Fig. 16)

les poteries est également à l'origine de la présence de fragments coquilliers dans les pâtes des céramiques découvertes en contexte archéologique.

Des blocs de pierre ont aussi pu être prélevés (Laporte 2009) ou débités sur l'estran pour servir de pavage ou de matériaux de construction.

Les mollusques marins ont différents prédateurs. S'ils peuvent être consommés par les oiseaux, c'est leur présence dans des contenus stomacaux de poissons qui a été mise en évidence sur un site médiéval de la Somme localisé à plus de $60 \mathrm{~km}$ de la mer (Figure 17). Leur présence a été identifiée dans une zone dépotoir au milieu des arêtes. Lors de la préparation des poissons ceux-ci ont été évidés et les viscères ont été jetés dans les poubelles, avec eux les milliers de coquillages dont ils étaient emplis.

Ces exemples archéologiques montrent que de tout temps l'homme a transporté avec lui des mollusques marins mais qu'il n'était pas toujours le moteur volontaire de ce transport.

\section{$\&$}

Dès la Préhistoire, l'Homme a différencié les mollusques marins qui contribuent à son alimentation des coquilles utilisées pour parer son corps ou orner des objets, voire des monuments. L'analyse archéomalacologique montre que cette sélection s'opère dès l'acquisition du mollusque. Ainsi, les coquilles échouées déjà dépourvues de la chair de 
l'animal seront préférées au recyclage des déchets alimentaires. Ces tests ont été sélectionnés pour leurs formes, pour leurs couleurs et ne sont pas toujours observés vivants car certains d'entre eux subsistent sous le niveau des plus basses mers. Ils sont sans doute, pour quelques uns d'entre eux, le symbole d'un monde étrange et mystérieux. La mythologie qui entoure le monde marin durant l'Antiquité l'atteste. Les coquilles ont cet avantage sur les coquillages de pouvoir voyager sans être altérées. Les distances qu'elles parcourent grâce au vecteur humain les chargent d'une valeur qui peut s'accroître en fonction de leur distance du lieu d'origine. Cette haute valeur ajoutée s'observe également à partir des coquillages alimentaires. Laccélération du transport des coquillages frais ou préparés avec le développement des moyens de locomotion, des voies de transport et des modes de conservation est aussi intéressante à observer en archéologie. En effet, elle ne se traduit pas par le transport systématique de tous les coquillages communément consommés par les populations côtières. Les mollusques mangés loin de la côte ne sont pas forcément les plus résistants à la dessiccation et aux fortes températures. Ce constat fait des coquillages alimentaires, au même titre que les coquilles « objets», des artefacts archéologiques chargés d'une composante culturelle.

La diffusion des coquilles et coquillages par l'homme est loin d'être entièrement contrôlée. Dès la Préhistoire, l'homme a déplacé ces organismes marins malgré lui en profitant de tout le panel offert par l'environnement marin (transport de matériau de construction, engrais, maintien au frais des aliments...). Si les distances parcourues par le passé restaient limitées, leur augmentation et l'accélération des transports ont permis aux coquillages vivants de coloniser d'autres territoires.

\section{NOTES}

Photo d'ouverture: Les coquilles de certains coquillages qui ont été consommés ou sont avariés sont parfois recyclées comme matériaux de construction comme ici dans les parcs ostréicoles (2011, île d'Oléron, Charente-Maritime, C. Dupont). 


\section{RÉFÉRENCES}

Beaune, S. A. 1995 Les hommes au temps de Lascaux 40000-10000 av. J.-C. Paris: Hachette.

Boislève, J., Labaune, F., Dupont, C. 2012 Décors peints à incrustations de coquillages en Armorique romaine, Aremorica. Études sur l'ouest de la Gaule romaine 5 : 9-32.

— 2011 Coquillages. Les décors marins d’Armorique, Archéologia 486: 25-35.

Cardon, D. 2003 Le monde des teintures naturelles. Paris: Belin.

- 1990 Guide des teintures naturelles. Plantes, Lichens, Champignons, Mollusques et Insectes. Neuchâtel, Suisse: Éditions Delachaux et Niestlé (Les guides du naturaliste).

Dupont, C. 2011 The Dog Whelk Nucella Lapillus and Dye Extraction Activities from the Iron Age to the Middle Ages along the Atlantic Coast of France, Journal of Island and Coastal Archaeology 6 (1): 3-23.

— 2006 La malacofaune de sites mésolithiques et néolithiques de la façade atlantique de la France: Contribution à l'économie et à l'identité culturelle des groupes concernés. Oxford: British Archaeological Reports, Archeopress (International Series 1571).

Dupont, C., Blondiau, L. 2006 Les coquillages marins du village antique de «La Ferme aux Mouches » (Pont-de-Metz, Somme) : d'une ressource alimentaire au témoin d'un artisanat? Revue du Nord 88 (368) : 173-179.

Dupont, C., Marchand, G. 2008 Coastal Exploitation in the Mesolithic of Western France: la Pointe SaintGildas (Préfailles), Environmental Archaeology 13 (2): 143-152.

Dupont, C., Marchand, G., Carrion, Y., et al. 2010 Beg-an-Dorchenn: une fenêtre ouverte sur l'exploitation du littoral par les peuples mésolithiques du sixième millénaire dans l'ouest de la France, Bulletin de la Société Préhistorique Française 107 (2) : 227-290.

Dupont, C., Tresset, A., Desse-Berset, N., et al. 2009 Harvesting the Seashores in the Late Mesolithic of North-Western Europe. A View from Brittany? Journal of World Prehistory 22 (2) : 93-111.

Garanger, J. 1992 La préhistoire dans le monde. Paris: Presse Universitaire de France.

Giot, P.-R., L'Helgouac'h, J., Monnier, J.-L. 1998 Préhistoire de la Bretagne, Évreux: Ouest-France.

Gruet, Y. 1993 Les coquillages marins: objets archéologiques à ne pas négliger: Quelques exemples d'exploitation et d'utilisation dans l'Ouest de la France, Revue archéologique de l'Ouest 10 : 57-161.

Gruet, Y., Bonnissent, D. 2002 Des coquilles Saint-Jacques (Pecten maximus) taillées avant d'être vendues aux pèlerins? Revue d’Archéométrie 26: 113-123.

Gruet, Y., Dupont, C. 2009 I - Les mollusques ou la malacofaune, B - Matériel et méthodes. C - Un ensemble malacologique de référence: Ponthezières. In L. Laporte (dir.) Des premiers paysans aux premiers métallurgistes sur les côtes charentaises. Chauvigny: Association des Publications Chauvinoises: 555-581.

Gruet, M., Lemonnier, L., Gruet, Y. 1999 VIII Les coquilles marines. In C. Burnez et P. Fouéré (eds.) Les enceintes néolithiques de Diconche à Saintes (Charente-Maritime). Mémoire de la Société Préhistorique Française 1998 (1) 139-145; 343-345.

Gutiérrez-Zugasti, I., Andersen, S.H., Araujo, A. C., et al. 2011 Shell Midden Research in Atlantic Europe: State of Art, Research Problems and Perspectives for the Future, Quaternary International: 70-85.

Hodder, I. 1993 The domestication of Europe. Oxford UK \& Cambridge USA: Blackwell.

Kayser, O. 1989 Le Mésolithique des îles bretonnes, Bulletin de l'AMARAI 2 : 1-2.

Laporte, L. (dir.) 2009 Des premiers paysans aux premiers métallurgistes sur les côtes charentaises. Chauvigny: Association des Publications Chauvinoises.

Mieszkowska, N., M.A., Kendall, S.J., Hawkins, R. et al. 2006 Changes in the Range of Some Common Rocky Shore Species in Britain - A Response To Climate Change? Hydrobiologia 555 : 241-251.

Pailler, Y., Stéphan, P., Gandois, H. et al. 2011 Évolution des paysages et occupation humaine en mer d'Iroise (Finistère, Bretagne) du Néolithique à l'âge du bronze, Norois 220 (2011/3) : 39-68.

Poppe, T., Goto, Y. 1991 European Seashells: Polyplacophora, Caudofoveata, Solenogastra, Gasteropoda. Germany : Verlag Christa Hemmen. 
- 1993 European Seashells : Scaphopoda, Bivalvia, Cephalopoda. Germany : Verlag Christa Hemmen.

Schulting, R. J. 2005 Comme la mer qui se retire : les changements dans l'exploitation des ressources marines du Mésolithique au Néolithique en Bretagne. Unité et diversité des processus de néolithisation sur la façade atlantique de l'Europe (vII-IV millénaires avant J.-C.), Mémoires de la Société Préhistorique Française 36 : 163-171.

— 1996 Antlers, Bone Pins and Flint Blades : the Mesolithic Cemeteries of Téviec and Hoëdic-Brittany, Antiquity $70: 335-350$.

Schulting, R., Tresset, A., Dupont C. 2004 From Harvesting the Sea to Stock Rearing Along the Atlantic Façade of North-Western Europe. Environmental Archaeology 9 : 143-154.

Taborin, Y. 1993 La parure en coquillage au Paléolithique. Paris : CNRS (Supplément à « Gallia préhistoire »). Wickham-Jones, C.R. 2003 The tale of the Limpet, British Archaeology 71 : 23.

\section{RÉSUMÉ}

Ne confondons pas coquilles et coquillages. Vision diachronique de l'archéologie des mollusques le long de la façade atlantique. Larrivée de l'archéomalacologie en tant que discipline nouvelle de l'archéologie ne cesse de mettre en évidence des utilisations parfois oubliées des coquillages le long du littoral atlantique français. Plusieurs activités nécessitent la collecte de l'animal vivant ou coquillage telles la consommation alimentaire ou l'extraction de colorant, d'autres n'utilisent que le test ou la coquille (parure, éléments de construction, décor mural, outil...). Tout au long de la chronologie, un même phénomène semble se répéter. Les coquilles des coquillages consommés sont rarement recyclées. Larchéologie permet de mettre en lumière cette dichotomie dès leur acquisition. Ainsi, la collecte du coquillage frais semble correspondre à une activité différente de celle qui consiste à ramasser des coquilles dépourvues de la chair de l'animal. Si certains mollusques marins autrefois consommés ne le sont plus actuellement, ce n'est pas forcément du fait d'une perte de leur accessibilité. Des phénomènes sociaux ou environnementaux ont pu effacer de notre mémoire des espèces de mollusques marins en tant que ressource alimentaire. La contrainte liée à la conservation de l'animal, le plus souvent frais a, par le passé, limité la diffusion de cette ressource alimentaire sur de longue distance. Ce facteur limitant n'est pas le seul à avoir exercé un tri parmi les espèces consommées loin du littoral. En effet, l'archéologie permet de comparer les ressources marines consommées par les populations inféodées aux zones côtières avec celles d'occupants plus continentaux. Ce ne sont pas forcément les mollusques, qui résistent le mieux à des séjours hors de l'eau de mer, qui seront les plus prisés par les populations les plus éloignées des gisements naturels. Enfin, les coquilles découvertes en contextes archéologiques sont parfois transportées par l'homme, involontairement, grâce à des vecteurs. Ceux-ci sont les témoins d'autres activités anthropiques intimement liées à l'environnement marin. 


\section{ABSTRACT}

Do not mix up shells and shellfish. A diachronic archaeological view of molluscs along the Alantic facade. The archaeomalacology is still a new discipline of the archaeology. It brings to light uses of shells sometimes forgotten along the French Atlantic coast. Several activities require the gathering of the alive animal like the food consumption or the extraction of dye, the others use only the test (ornament, elements of construction, wall decoration, tool). Throughout the chronology, the same phenomenon repeats. The shells of the consummate shellfish are rarely recycled. The archaeology allows to bring to light this dichotomy from their acquisition. So, the gathering of the fresh shell seems to correspond to a different activity from the one which consists in collecting shells devoid of some flesh. If certain marine mollusks formerly consummate are not eaten any more at present, it is not only linked to a loss of their accessibility. Social or environmental phenomena were able to erase species of marine mollusks as food resource of our memory. The constraint connected to the preservation of the animal, mostly freshly, limited in the past, the distribution of this food resource on long distance. This limiting factor is not the only one to have exercised a sorting among the species consumed far from the coast. Indeed, the archaeology allows to compare the marine resources consumed by the populations vassal of the coastal zones of those occupying more continental territories. It is not necessarily the mollusks which resist best stays outside the sea water, which will be the most appreciated by the populations the most distant from the natural deposits. Finally, shells discovered in archaeological contexts are sometimes involuntarily transported by the man by means of vectors. These last ones are witnesses of other anthropological activities confidentially connected to the marine environment.

\section{MOTS CLÉS}

Coquillage, coquille, archéologie, nourriture, parure, teinture, décor mural, animal, matière première, façade atlantique française

\section{KEYWORDS}

Shellfish, shell, archaeology, food, ornament, dyeing, shell inlays, animal, raw material, French Atlantic façade 